\title{
Nonlinear microscopy of lipid storage and fibrosis in muscle and liver tissues of mice fed high-fat diets
}

\author{
Christian Brackmann \\ Chalmers University of Technology \\ Department of Chemical and Biological Engineering \\ Molecular Microscopy \\ 41296 Göteborg, Sweden
}

\section{Britt Gabrielsson}

Chalmers University of Technology

Department of Chemical and Biological Engineering

Food Science

41296 Göteborg, Sweden

\section{Fredrik Svedberg}

Chalmers University of Technology

Department of Chemical and Biological Engineering

Molecular Microscopy

41296 Göteborg, Sweden

\author{
Agneta Holmäng \\ Göteborg University \\ The Sahlgrenska Academy \\ Institute of Neuroscience and Physiology \\ Department of Physiology \\ 40530 Göteborg, Sweden
}

\section{Ann-Sofie Sandberg}

Chalmers University of Technology

Department of Chemical and Biological Engineering

Food Science

41296 Göteborg, Sweden

\section{Annika Enejder}

Chalmers University of Technology

Department of Chemical and Biological Engineering

Molecular Microscopy

41296 Göteborg, Sweden

\begin{abstract}
Hallmarks of high-fat Western diet intake, such as excessive lipid accumulation in skeletal muscle and liver as well as liver fibrosis, are investigated in tissues from mice using nonlinear microscopy, second harmonic generation (SHG), and coherent anti-Stokes Raman scattering (CARS), supported by conventional analysis methods. Two aspects are presented; intake of standard chow versus Western diet, and a comparison between two high-fat Western diets of different polyunsaturated lipid content. CARS microscopy images of intramyocellular lipid droplets in muscle tissue show an increased amount for Western diet compared to standard diet samples. Even stronger diet impact is found for liver samples, where combined CARS and SHG microscopy visualize clear differences in lipid content and collagen fiber development, the latter indicating nonalcoholic fatty liver disease (NAFLD) and steatohepatitis induced at a relatively early stage for Western diet. Characteristic for NAFLD, the fibrous tissue-containing lipids accumulate in larger structures. This is also observed in CARS images of liver samples from two Western-type diets of different polyunsaturated lipid contents. In summary, nonlinear microscopy has strong potential (further promoted by technical advances toward clinical use) for detection and characterization of steatohepatitis already in its early stages. (C) 2010 Society of Photo-Optical Instrumentation Engineers. [DOI: 10.1117/1.3505024]
\end{abstract}

Keywords: microscopy; non-linear optics; liver; skeletal muscle.

Paper 10210R received Apr. 21, 2010; revised manuscript received Aug. 15, 2010; accepted for publication Sep. 13, 2010; published online Nov. 30, 2010.

\section{Introduction}

Lipid storage in nonadipose tissues is known to be related to insulin resistance and predisposes the development of type- 2 diabetes and cardiovascular disease. ${ }^{1}$ Increased levels of intracellular lipids in skeletal muscle have been shown to associate with insulin resistance in sedentary subjects. ${ }^{2,3}$ Furthermore, obese subjects are prone to development of nonalcoholic fatty liver disease (NAFLD), which in turn can progress to nonalcoholic steatohepatitis (NASH), a fatty inflammation of the liver that increases the risk for future complications due to fibrotic scarring of the liver. ${ }^{4,5}$ Histochemical analysis of liver biopsies is so far the only way to confirm the diagnosis of NASH, and to assess the degree of fibrosis of the liver. ${ }^{6}$

Address all correspondence to: Annika Enejder, Chalmers University of Technology, Department of Chemical and Biological Engineering, Molecular Microscopy, 41296 Göteborg, Sweden. Tel: 46-31-772-38-52; Fax: 46-31-772-38-01. E-mail: enejder@chalmers.se.
Biochemical analysis can provide reliable average measures on tissue triglyceride content, whereas microscopy offers detailed visual information on distribution and morphology of lipid stores on a cellular scale. Traditionally, this relies on sample staining, ${ }^{7}$ but electron microscopy has also been used for intracellular lipid visualization. ${ }^{3}$ However, histological staining introduces uncertainties depending on the efficiency of the marker to attach to the lipid structures. In addition, bright-field microscopy has limited axial resolution and requires thin samples to avoid problems with absorption, multiple scattering, and depolarization, all limiting factors for studies of thicker tissue samples.

Laser-based nonlinear microscopy is emerging as a powerful research tool for molecular-specific imaging of cells and tissue without having to rely on sample labeling, thus also fresh tissues can be studied. The nonlinear signal generation is limited to the

$1083-3668 / 2010 / 15(6) / 066008 / 10 / \$ 25.00$ C 2010 SPIE 
laser beam focal volume, resulting in high spatial resolution in three dimensions. Excitation using near-infrared wavelengths reduces risks for photochemical effects and damages, and it also provides good sample penetration, which permits imaging with optical sectioning of relatively thick samples. Thus, nonlinear microscopy is very suitable for tissue characterization.

Second harmonic generation (SHG) microscopy is a nonlinear technique able to specifically visualize structures of noncentrosymmetric molecular arrangement. The SHG process is induced by illumination with a high-intensity laser beam, where photons having twice the energy of the excitation light are generated. The method is well characterized ${ }^{8}$ and suitable for imaging of ordered structural proteins such as myosin and collagen. 9 The ability to image myosin makes SHG microscopy a good complement to conventional optical microscopy and electron microscopy for characterization of muscle tissue structure. ${ }^{10,11}$ In addition, the ability to detect collagen, which is accumulated in fibrotic liver, makes it suitable for detection and analysis of liver fibrosis. ${ }^{12,13}$

Another nonlinear technique, coherent anti-Stokes Raman scattering (CARS) microscopy, ${ }^{14,15}$ has developed into a valuable tool for label-free, selective imaging of lipids. Chemical specificity is achieved by probing inherent molecular vibrations, in the case of lipids usually the symmetric stretch vibration of $\mathrm{CH}_{2}$ groups in the acyl chains. A number of publications on investigations of intracellular lipids using CARS microscopy has emerged during the last few years, ${ }^{16-19}$ and the technique has also been applied for monitoring hepatic lipid content. ${ }^{20}$ Of special interest is that combined SHG and CARS microscopy of liver tissue allows for simultaneous imaging of fibrosis and lipid storage, two phenomena coupled in the development of NAFLD. In addition, combination of the methods is also valuable for studies of lipid accumulation in skeletal muscle tissue.

The aim of this study was to investigate whether multimodal nonlinear microscopy could be used as a tool to study the effects of high-fat diet intake on skeletal muscle and liver tissue of mice. Lipid contents of muscle and liver tissue as well as liver fibrosis were visualized using simultaneous CARS and SHG microscopy on specimens from wild-type C57BL/6J mice fed standard rodent chow or a Western diet with high-fat content. Second, the effect of a high-fat/sucrose diet (HFSD), supplemented with either minced beef or minced herring fillets, on muscle and liver lipid storage in low density lipoprotein receptor $(L d l r)$-deficient mice was investigated by means of CARS microscopy. The phenotype of the Ldlr-deficient mouse on standard chow is mildly elevated plasma low-density lipoprotein (LDL) levels, whereas addition of cholesterol to a high-fat diet increases plasma very low-density lipoprotein (VLDL) levels and consequently accelerates the development of atherosclerotic plaques. ${ }^{21}$

\section{Materials and Methods}

\subsection{Animals and Diet}

The study was approved by the local Animal Ethics Committee at University of Gothenburg, Gothenburg, Sweden.

\subsubsection{Experiment 1}

Seven-week-old male C57BL/6J mice obtained from Charles River Laboratories (Sulzfeld, Germany) were kept under standardized conditions with free access to water and chow; laboratory lights were on between 7 a.m. and 7 p.m. The mice were randomly assigned to two groups ( $n=6$ per group) given either standard rodent chow or a Western diet (Harlan TD.88137; Harlan Laboratories, Boxmeer, the Netherlands) for up to 12 weeks. The energy density was $3.8 \mathrm{kcal} / \mathrm{g}$ (fat, $4.2 \mathrm{E} \%$; carbohydrates, $70.8 \mathrm{E} \%$; proteins, $25.0 \mathrm{E} \%$ ) for the standard rodent chow, and $4.5 \mathrm{kcal} / \mathrm{g}$ (fat, $42 \mathrm{E} \%$; carbohydrates, 42.7 $\mathrm{E} \%$; proteins, $15.2 \mathrm{E} \%$ ) for the Western diet that also contained $0.2 \%$ cholesterol. Two mice from each diet group were killed after 4, 8, and 12 weeks. Skeletal muscle tissues (soleus and diaphragm) and pieces of liver were placed in phosphate buffered saline (PBS) (approximately $30^{\circ} \mathrm{C}$ ) for subsequent SHG/CARS microscopy. Pieces of liver were also frozen in liquid nitrogen for lipid staining by Oil Red $\mathrm{O}$ in frozen sections (Histo-Center AB, Göteborg, Sweden).

\subsubsection{Experiment 2}

Seven-week-old male Ldlr-deficient mice (Jackson Laboratories, stock number 002207) were obtained from Charles River Laboratories (Sulzfeld, Germany). After one week of acclimatization to the conditions in our animal facility (see before), the mice ( $n=14$ per group) were randomly assigned to receive either a high fat/high sucrose diet supplemented with minced beef (HFSD beef) or with minced herring (HFSD herring). The diets contained $21.5 \%$ fat and $0.074 \%$ cholesterol (wt/wt), and the diet fat compositions grouped in categories of saturated, monounsaturated, and polyunsaturated lipids were $66.3,29.1$, and $2.6 \%$, respectively, for HFSD beef, and 60.1, 29.3, and 8.0\%, respectively, for HFSD herring. After 16 weeks of diet the mice were killed by an overdose of sodium pentobarbital during three consecutive days, and samples of the diaphragm and one liver lobe were collected and placed in PBS (approximately $30^{\circ} \mathrm{C}$ ) for subsequent CARS microscopy.

\subsection{Nonlinear Microscopy}

The experiments are summarized in Fig. 1, which also shows the experimental setup used for nonlinear microscopy. The laser system consists of a Nd:Vanadate pump laser [HighQ (Hohenems, Austria) picoTRAIN], and two ring-cavity optical parametrical oscillators (OPO), APE (Berlin, Germany) Levante. The pump laser emits pulses of 7-ps duration and wavelength of $1064 \mathrm{~nm}$ at a repetition rate of $76 \mathrm{MHz}$. The total average output power of the pump laser is $10 \mathrm{~W}$ split into three beams, one directly aligned into the microscope and one beam directed to each OPO. The OPOs provide tunable radiation covering the wavelength intervals 785 to $845 \mathrm{~nm}$ and 855 to $920 \mathrm{~nm}$, with average output powers in the range 0.3 to $1.0 \mathrm{~W}$. The 1064-nm and OPO beams are aligned into a common optical path using dichroic mirrors, and temporal overlap is achieved by means of individual delay lines for the OPO beams. The copropagating beams are directed into the microscope (Nikon TE-2000E) beam scanning unit and focused on the sample by an objective $(40 \times$ Nikon Plan Fluor NA 1.3 or $60 \times$ Nikon TIRF NA 1.49). Typical average powers at the sample are $50 \mathrm{~mW}$ for the OPO beams and $30 \mathrm{~mW}$ for the 1064 beam. CARS and SHG are generated in the high-intensity region of the diffractionlimited focal volume of the laser beams, and the signals 


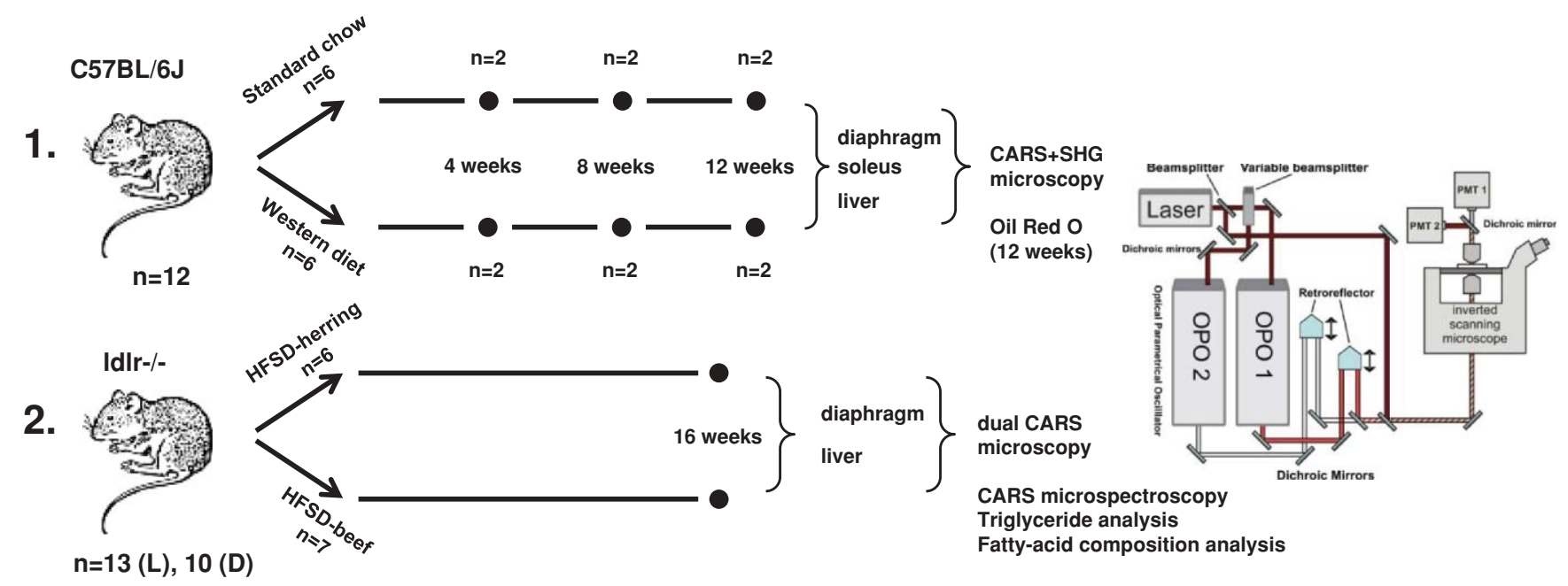

Fig. 1 Summary of the experiments carried out on skeletal muscle and liver tissues of mice. In experiment 1 with 12 mice, intake of standard chow and a Western-type high-fat diet was compared during 12 weeks by SHG and CARS microscopy. Experiment 2 with 28 mice, from which 13 liver and 10 diaphragm samples were characterized by CARS microscopy, compared the differences between two high-fat diets with different levels of polyunsaturated lipids (see Sec. 2 for further details). The experimental setup for nonlinear microscopy, shown to the right, consists of a pump laser, two OPOs, and an inverted microscope with laser beam scanner. The generated signals are collected in the forward direction, separated by a dichroic mirror, and detected by photomultiplier tubes (PMTs).

propagating in the forward direction are collected by an aspherical lens (NA 0.8) separated by a beamsplitter and directed to one photomultiplier tube each (Hamamatsu PMC100-1/PMC100 20). Bandpass filters are used to isolate the signals and suppress background light, in particular residual laser radiation.

Combined SHG and CARS microscopy (Experiment 1) or dual CARS microscopy ${ }^{22}$ (Experiment 2) measurements were carried out on samples of liver and skeletal muscle tissue of mice. For combined SHG and CARS measurements, a single OPO tuned to $817.0 \mathrm{~nm}$ was used that—combined with the 1064-nm beam-excites the $\mathrm{CH}_{2}$ symmetric stretch vibration at wave number $2845 \mathrm{~cm}^{-1}$, and the generated CARS signal is detected at $663 \mathrm{~nm}$. The 817-nm beam also induces SHG detected at $409 \mathrm{~nm}$. To obtain quantitative information on lipid storage from CARS microscopy measurements carried out in Experiment 2, a dual CARS arrangement was used to simultaneously measure the nonresonant background and be able to subtract it from the $\mathrm{CH}_{2}$ CARS signal. For this purpose, the short-wavelength OPO remained tuned to $817 \mathrm{~nm}\left(2845 \mathrm{~cm}^{-1}\right)$, whereas the longwavelength OPO was tuned to $863.6 \mathrm{~nm}$. Combined with the 1064-nm beam, the latter generates a CARS process probing wave number $2185 \mathrm{~cm}^{-1}$ in the so-called silent Raman region, where biological tissue lacks vibrational resonances and the nonresonant background can be measured.

Fresh tissue specimens were manually cut in slices a few hundred microns thick, and carefully mounted between microscopy cover slides. Tissue specimens of soleus muscle, diaphragm, and liver were collected from wild-type mice fed either standard chow or Western diet and from Ldlr-deficient mice fed HFSD herring or beef. The soleus muscle is of interest due to its relatively high amount of oxidative muscle fibers. The flat geometry of the diaphragm simplifies the sample preparation and makes microscopy of it relatively straightforward.

Images were collected either as single planes or as z-stacks covering 30 to 60 image planes and forming 3-D images. The scanned image area ranged from $20 \times 20 \mu \mathrm{m}^{2}$ to $200 \times$ $200 \mu \mathrm{m}^{2}$, with larger fields of view $\left(140 \times 140 \mu \mathrm{m}^{2}\right.$ and $200 \times$
$200 \mu \mathrm{m}^{2}$ ) used for the analysis of liver samples, and smaller fields of view $\left(40 \times 40 \mu \mathrm{m}^{2}\right)$ used for the analysis of muscle tissue. The numbers of measurements on soleus, diaphragm, and liver specimens are summarized in Table 1.

CARS spectra of liver tissues from a HFSD-herring and HFSD-beef mouse were obtained by collecting CARS microscopy images with the OPO tuned to wavelengths between 807 and $824 \mathrm{~nm}$, probing vibrations between wave numbers 2740 and $3000 \mathrm{~cm}^{-1}$. At each wavelength an image of lipid stores in the liver tissue and a reference image in the cover slide glass, to normalize for varying excitation efficiency, were registered. From the series of CARS microscopy images, the normalized lipid droplet CARS signal was averaged and plotted versus probed wave number to obtain a CARS spectrum.

\subsection{Image Analysis}

Nonlinear microscopy images were segmented into binary images for further analysis. For lipid droplet evaluation, the binary data obtained from the CARS microscopy images were analyzed using two tools of the software ImageJ, ${ }^{23}$ the built-in particle analyzer, and a 3-D object counter plug-in routine. Particle sizes evaluated as areas and volumes were recalculated into equivalent diameters. Relative areas of tissue lipid content and liver fibrosis, visualized in the CARS and SHG images, respectively, were calculated as the number of thresholded pixels divided by the total number of pixels. For the dual CARS measurements carried out in Experiment 2, the measured nonresonant background was subtracted, resulting in a compensated CARS signal essentially consisting of the $\mathrm{CH}_{2}$ vibrational resonant contribution.

\subsection{Tissue Biochemical Analysis}

Frozen liver biopsies (weighing 100 to $200 \mathrm{mg}$ ) were homogenized in ten volumes of PBS ( $\mathrm{pH}$ 7.4). The homogenates were weighed before and after removal of approximately 100- $\mu 1$ aliquot for triglyceride analysis, and the remainder of the 
Brackmann et al.: Nonlinear microscopy of lipid storage and fibrosis in muscle and liver tissues ...

Table 1 Numbers of samples and SHG/CARS microscopy measurements for the two studies carried out on skeletal muscle and liver tissues of mice.

\begin{tabular}{|c|c|c|c|c|c|c|c|}
\hline & Mouse category & $\begin{array}{c}\text { Soleus } \\
\text { samples }\end{array}$ & $\begin{array}{l}\text { Soleus } \\
\text { data }\end{array}$ & $\begin{array}{l}\text { Diaphragm } \\
\text { samples }\end{array}$ & $\begin{array}{l}\text { Diaphragm } \\
\text { data }\end{array}$ & Liver samples & Liver data \\
\hline \multirow[t]{2}{*}{ Experiment 1} & $\begin{array}{c}\text { Wild-type; standard } \\
\text { diet }\end{array}$ & 6 & $\begin{array}{l}9 \text { images } \\
17 \text { z-stacks }\end{array}$ & - & - & 2 & 5 z-stacks \\
\hline & $\begin{array}{c}\text { Wild-type; Western } \\
\text { diet }\end{array}$ & 6 & $\begin{array}{l}18 \text { images } \\
26 \text { z-stacks }\end{array}$ & - & - & 2 & 3 z-stacks \\
\hline \multirow[t]{2}{*}{ Experiment 2} & $\begin{array}{l}\text { Ldllr-deficient; } \\
\text { HFSD-beef }\end{array}$ & - & - & 4 & 35 images & 7 & 34 images \\
\hline & $\begin{array}{l}\text { Ldlr-deficient; } \\
\text { HFSD-herring }\end{array}$ & - & - & 6 & 30 images & 6 & 29 images \\
\hline
\end{tabular}

homogenate was freeze-dried. The remaining homogenate was freeze-thawed for three cycles to disrupt the cells, and total triglyceride content was determined enzymatically following manufacturer protocol (T2449, F6428; Sigma-Aldrich, Stockholm, Sweden). Results were back-calculated and expressed as milligram triglycerides per liver dry weight.

\section{Results and Discussion}

\subsection{Skeletal Muscle Tissue; Structure and Intramyocellular Lipid Content}

Figure 2 shows pairs of simultaneously measured SHG (left column) and CARS (middle column) microscopy images in soleus muscle for mice fed standard rodent chow [Fig. 2(a)] and Western diet [Figs. 2(b) and 2(c)]. The ordered arrangement of myosin molecules in the muscle filaments induces birefringence and makes them SHG-active. Thus, the cross-striation pattern of muscle tissue A-bands, also recognizable from polarization microscopy of sectioned tissue,${ }^{24}$ can be visualized by SHG microscopy. Intramyocellular lipid droplets, imaged by CARS microscopy and typically $1 \mu \mathrm{m}$ in diameter, can be seen positioned longitudinally along the muscle fibers and located in-between the myofibrils. This is clearly shown in the overlay CARS/SHG images (right column), and in particular in the images of Fig. 2(c) covering a smaller field of view. The observed lipid droplet localization is in agreement with observations reported for human skeletal muscle tissue, ${ }^{25}$ and recent studies have shown that the intramyocellular lipid droplets, forming a cellular energy source, are located between the intermyofibrillar mitochondria and to a larger extent in the oxidative, mitochondria-rich type-1 fibers than type 2. ${ }^{26,27}$ Approximately $40 \%$ of the muscle fibers in soleus from male $\mathrm{C} 57 \mathrm{BL} / 6 \mathrm{~J}$ mice are of the slow-twitch type $1 .{ }^{28}$ Combining two-photon fluorescence of labeled mitochondria ${ }^{26}$ with CARS and SHG microscopy would permit colocalization of all structures of interest, and this is a topic to be investigated further.

CARS microscopy on skeletal muscles (soleus and diaphragm) from wild-type mice fed standard or Western diet generally showed low content of lipids, irrespective of diet. After four weeks, intramyocellular lipid droplets were detected in one third of the images measured in the soleus sample of one mouse fed Western diet, and a sample image is shown in Fig. 2(c). Measurements on soleus samples from the three other mice investigated at this time point, one fed Western diet and two fed standard chow, showed no intramyocellular lipid content. Diaphragm samples investigated after four weeks of diet intake showed no lipid content and neither did additional measurements carried out on samples of extensor digitorum longus or gastrocnemius (data not shown). After eight weeks, approximately one third of the CARS microscopy images measured in one of the two investigated Western diet soleus samples showed lipids. The other soleus data, measured in the other Western diet sample and two standard diet samples, showed no intramyocellular lipids. Measurements carried out after 12 weeks showed lipid droplets in soleus samples of all four mice investigated, i.e., for both standard chow and Western diet [Figs. 2(a) and 2(b)]. Nevertheless, Oil red $\mathrm{O}$ staining of soleus samples showed
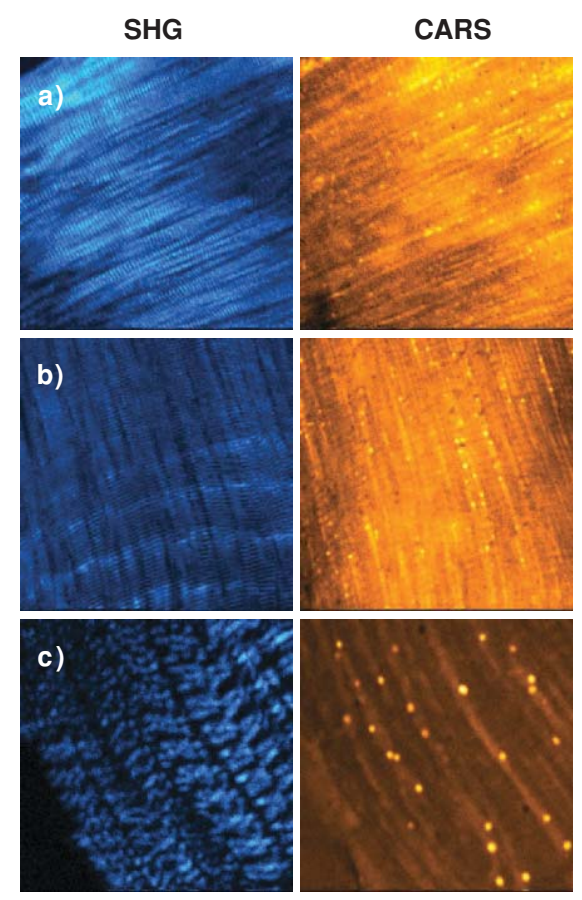

CARS
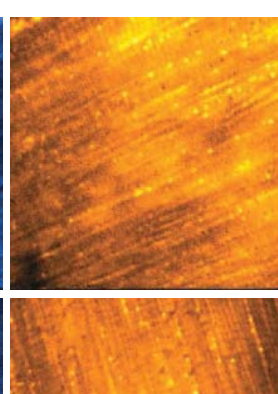

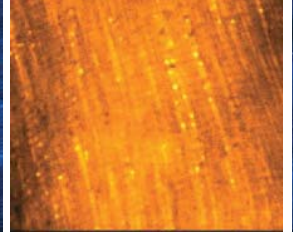

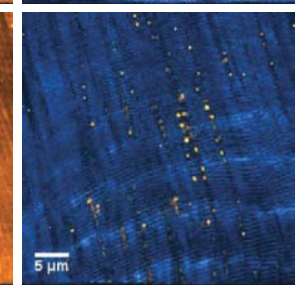

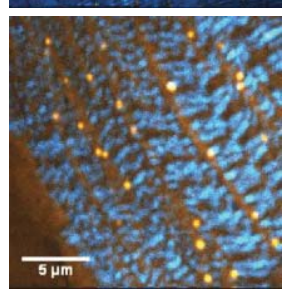

Fig. 2 Muscle tissue structure and intramyocellular lipid droplets imaged in soleus muscle by simultaneous SHG and CARS microscopy. Intramyocellular lipid droplets, monitored by CARS microscopy, were detected both in samples of mice fed (a) standard rodent chow and (b) Western diet for 12 weeks. The lipid droplets are aligned along the muscle fibers and located between the myofibrils, clearly seen in images (c) (measured after 4 weeks of Western diet) covering a smaller field of view. 
an overall low lipid content for all these specimens (data not shown). The diaphragm samples measured at this time point showed lipid droplets in one Western diet and one standard chow specimen. In summary, the measurements indicate overall low amounts of intramyocellular lipid droplets; nevertheless, more of the CARS microscopy images measured in Western diet samples showed lipid droplets compared to standard chow. In agreement, high-fat diet intake has been reported to increase intramyocellular lipid levels in humans. ${ }^{3}$ However, more in-depth investigations are required to understand the coupling between diet fat content and lipid storage in skeletal muscle tissue.

\subsection{Influence of High-Fat Western Diet on Hepatic Lipid Content}

A comparison between hepatic lipid storage in mice fed standard chow or Western diet for 12 weeks is presented in Fig. 3. Lipid contents of liver specimens from four mice, two from each diet group, have been analyzed using both CARS microscopy (left column images) and histochemical staining with Oil Red O (right column images). In the CARS images the strongest signals (in bright yellow) were obtained from lipid droplets located in a matrix of hepatocytes visualized in the background (red).

Although the specificity that can be obtained with CARS microscopy for visualization of lipids is well known, the complex composition of liver tissue motivates a comparison with conventional methods, and Oil Red O staining of liver sections from the same animals was compared with the results from CARS microscopy. Several staining protocols for different types of lipids exist, ${ }^{29}$ and using the Oil Red O marker is an established method for staining of lipids in general. It is therefore a suitable choice for visualization of overall lipid storage in the liver tissue, and for comparison with CARS microscopy that visualizes lipids probing $\mathrm{CH}_{2}$ groups. Nevertheless, for detailed analysis of the hepatic lipid contents, more specific staining alternatives may be appropriate.

Oil Red O staining and CARS microscopy showed lipid distributions with good overall agreement for all individuals with low and high hepatic lipid contents for mice fed standard and Western diets, respectively. CARS microscopy images measured in liver tissues from mice fed the standard diet revealed an interindividual variation in hepatic lipid storage, showing a distribution of small lipid droplets for one individual [Fig. 3(a)] and a liver tissue matrix containing only a few lipid droplets for the other individual [Fig. 3(b)]. This was in line with the results obtained by the Oil Red $\mathrm{O}$ analysis, revealing low amounts of stained structures, in particular for the specimen of Fig. 3(b). Still, the hepatic lipid content was significantly lower in mice fed the standard diet compared with those given the Western diet [Figs. 3(c) and 3(d)], where much larger lipid structures could be observed with both CARS microscopy and Oil Red O staining. Additionally, the distributions of lipid structures in the livers of these mice show a high degree of heterogeneity.

Average lipid droplet diameters and the relative area covered by lipid structures were evaluated from the CARS microscopy images. The large number of small lipid droplets in the liver samples resulted in average diameters of $3 \mu \mathrm{m}$ for both diets. However, the size of the largest lipid structures imaged in the Western diet samples was approximately twice the size of those in the standard chow samples. The evaluated maximum diameter averaged over three standard and four Western diet images was 16 and $34 \mu \mathrm{m}$, respectively.

The average relative lipid area, presented in Fig. 3(e), was approximately three times higher for the Western diet mice compared to the standard diet mice with values of 13 and $4 \%$ of the scanned area, respectively. The larger spread in the relative lipid area obtained in liver tissues from mice fed the Western diet reflects the heterogeneity in lipid distribution for these livers, which can be observed in the CARS and Oil Red O images of Figs. 3(c) and 3(d).

The SHG microscopy images recorded simultaneously with the CARS images in the same field of view are shown in the middle column in Figs. 3(a) through 3(d). All four images contain SHG-generating fibrous structures, identified as collagen in previous studies of liver tissue. ${ }^{12,13}$ The detection of collagen was also confirmed by comparative measurements with van Gieson staining and SHG microscopy on a liver sample from a mouse fed a high-fat diet. The results are shown in Fig. 4 with very good agreement between the collagen stained in pink [Fig. 4(a)] and the structures visualized by SHG microscopy in the same region [Fig. 4(b)]. The high spatial resolution of SHG microscopy and the negligible background makes it possible to discern fine collagen structures difficult to distinguish in the staining image, for example the thin fibers indicated in Fig. 4(b). Thus, with the ability to measure fresh, unstained samples without sectioning SHG microscopy is clearly a suitable method for specific imaging of collagen in liver tissue.

Comparing the SHG images of Fig. 3, livers from mice fed a standard diet showed a few thin structures, whereas livers from mice fed the Western diet contained entangled networks of thicker fibers, indicating development of liver fibrosis. A pattern of fibers surrounding the hepatocytes can be seen, which is in agreement with the arrangement observed in histochemical images of liver tissue with developed steatohepatitis. ${ }^{30}$

Figure 3(f) shows the relative fiber area evaluated from the SHG microscopy images. Although data of both groups show spread values, the highest values, above $\sim 25 \%$, are obtained for the Western diet specimens with an average value approximately three times higher, $4.8 \%$ compared to $1.5 \%$ of the image area, than that for the standard diet specimens.

Previous studies in mice have shown that fiber formation in the liver is detected only after a relatively long time of highfat feeding. ${ }^{30}$ For example, DeLeve et al. reported developed steatohepatitis in mice after 6 and $9 \frac{1}{2}$ months. ${ }^{31}$ Approximately $0.1 \%$ (standard control diet), $0.4 \%$ (Western diet, 6 months), and $5 \%$ (Western diet, $9 \frac{1}{2}$ months) of the liver area was covered by Sirius Red-stained collagen fibers. Thus, their $9 \frac{1}{2}$ month data correspond to numbers obtained from SHG microscopy data already after 12 weeks, as shown in the present study. However, directly comparing the degree of steatohepatitis from data obtained by means of different microscopy techniques is questionable. As mentioned previously, results obtained with histochemical methods have an uncertainty related to the staining efficiency, and can underestimate the development and extent of liver fibrosis. We show that progression of liver fibrosis can be studied in diet-induced obese mice using SHG microscopy after 12 weeks of Western diet. The high sensitivity of the SHG technique for collagen detection is well known, ${ }^{9}$ as well as the higher spatial resolution achieved compared to staining methods ${ }^{12,32}$ (Fig. 4). This makes SHG microscopy suitable for early 

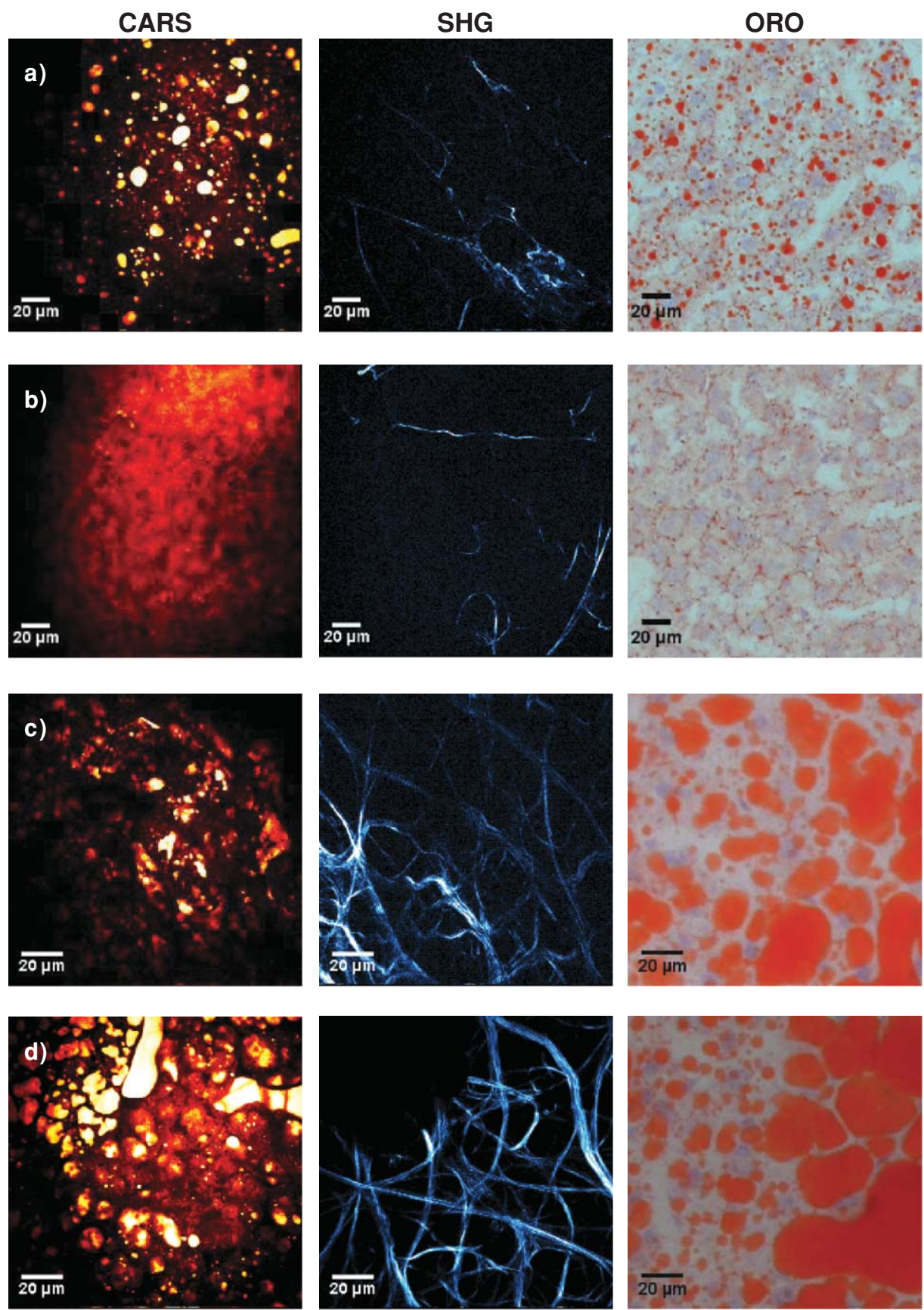

e)

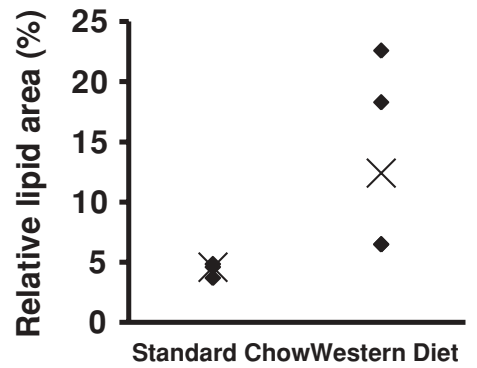

f)

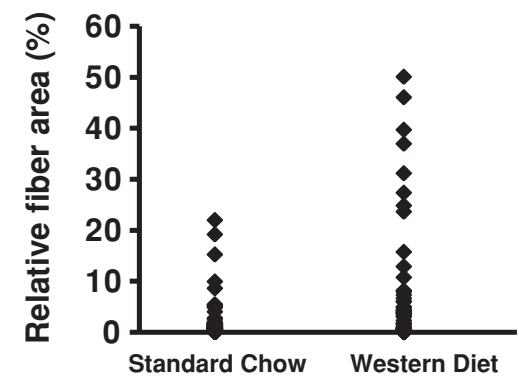

Fig. 3 (a) through (d) show CARS, SHG, and Oil Red O staining (ORO) microscopy images of liver tissues from wild-type mice fed standard or Western diets. The CARS microscopy images show stored lipids, the SHG images visualize collagen fiber distributions, and the Oil Red O stains hepatic lipids, verifying the CARS technique. Good agreement can be seen between the lipid distributions visualized using CARS microscopy and staining both for standard diet [(a) and (b)] and Western diet [(c) and (d)] mice. The SHG images clearly show an increased amount of fibrous structures, signifying developed liver fibrosis for the mice fed Western diet for 12 weeks [(c) and (d)]. The liver lipid content and the degree of liver fibrosis have been quantified as relative image areas covered by (e) lipid structures and ( $\mathrm{f}$ ) collagen fibers, respectively. The Western diet specimens typically show three times higher amounts of hepatic lipid content and three times higher fibrous content. 
detection of steatohepatitis. Furthermore, recent developments in optical fiber technology and endomicroscopy are beneficial for studies in vivo and also for future clinical use. ${ }^{33,34}$

\subsection{Influence of Diet Fatty Acid Composition on Hepatic Lipid Content}

It has been suggested that reduced amounts of hepatic long-chain polyunsaturated fatty acids contribute to NAFLD in humans, ${ }^{35}$ and in particular the marine polyunsaturated fatty acids EPA and DHA. ${ }^{36,37}$ Indeed, deficiency of essential fatty acids, has long been used in animal models to study the development of hepatic steatosis. ${ }^{38}$ Related to this, livers from $L d l r$-deficient mice fed HFSD beef or herring were analyzed by dual CARS microscopy. Since the HFSD herring contained larger amounts of long-chain polyunsaturated fatty acids, the aim was to investigate whether qualitative and quantitative differences in hepatic lipid storage could be detected by CARS microscopy.
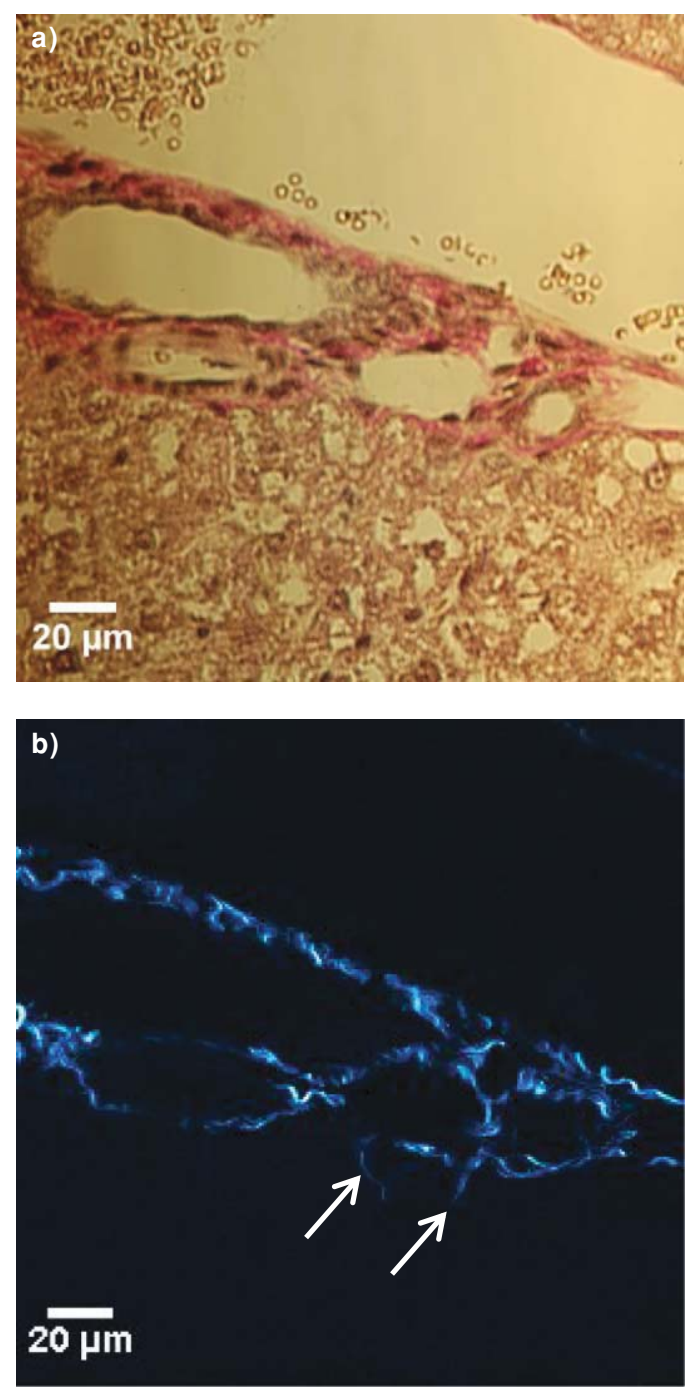

Fig. 4 (a) Staining and (b) SHG microscopy images of liver tissue from a mouse fed high-fat Western diet. The collagen stained pink in the tissue in (a) matches the structure visualized by SHG microscopy in (b). The SHG image is background-free and details difficult to distinguish with staining can be visualized, exemplified by the thin fibers indicated by arrows in (b).
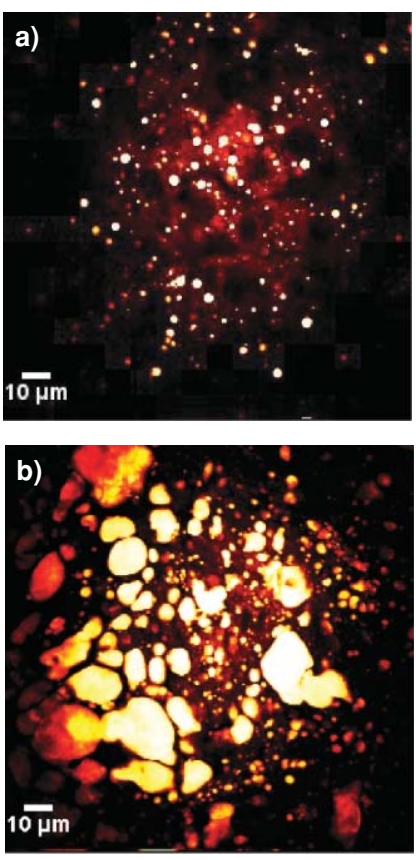

c)

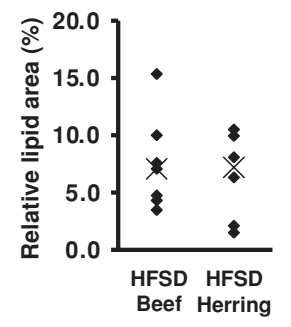

d)

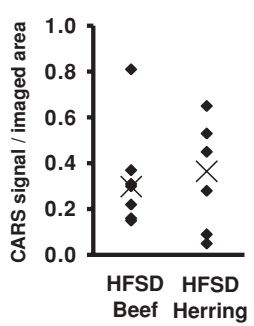

e)

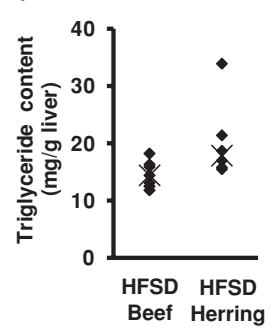

Fig. 5 CARS microscopy of liver tissue from $L d l r$-deficient mice fed HFSD beef or herring. Tissues of (a) low and (b) high lipid content were found in both diet groups. The liver lipid content, quantified from CARS images as (c) relative lipid area and (d) normalized CARS signal per area, is presented together with (e) the liver triglyceride content measured by biochemical analysis. A slightly higher amount of lipids can be observed in liver tissues of the HFSD-herring category $[(\mathrm{d})$ and $(\mathrm{e})]$

Figure 5 summarizes the results obtained comparing the two diets. On an individual basis, mice fed either diet showed varying liver lipid contents, exemplified by the images in Fig. 5(a) (low lipid content) and Fig. 5(b) (high lipid content). Quantitative results obtained from CARS microscopy and triglyceride analysis of liver tissues from mice fed both diets (Table 1 are presented in Figs. 5(c), 5(d), and 5(e). The average lipid droplet diameter was approximately $4 \mu \mathrm{m}$ for both HFSD-herring and HFSD-beef mice, with a difference on the order of the spatial resolution of the images $(0.55 \mu \mathrm{m})$. The maximum evaluated diameters were 36 and $42 \mu \mathrm{m}$ for samples from HFSD-herring and HFSD-beef mice, respectively.

Comparison of the relative lipid areas, i.e., the density of lipid droplets in the liver tissues, generally showed somewhat higher values for the HFSD-herring diet group, suggesting a higher amount of lipids stored in the liver. This was supported by the biochemical analysis of hepatic triglyceride content presented in Fig. 5(e), showing a $24 \%$ higher median triglyceride level in livers from the HFSD-herring mice. Quantification of the lipid CARS signal is complex due to the unspecific nonresonant 


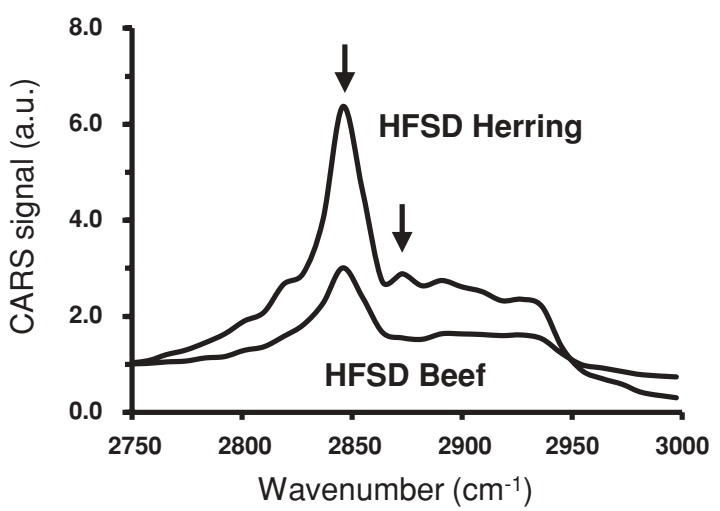

Fig. 6 CARS spectra of lipid droplets in liver tissue from $L d l r$-deficient mice fed HFSD beef or herring. Both spectra show a distinctive peak at $2850 \mathrm{~cm}^{-1}$ (upper arrow), corresponding to the $\mathrm{CH}_{2}$ symmetric stretch vibration. Differences can be seen in the signal strengths of the $\mathrm{CH}_{2}$ peak and the 2900- to $2950-\mathrm{cm}^{-1}$ region relative to the nonresonant background level at $2750 \mathrm{~cm}^{-1}$. The HFSD-herring spectrum also shows an additional small peak around $2880 \mathrm{~cm}^{-1}$ (lower arrow); for interpretation, see Sec. 3.

signal contribution that forms a background and also couples to the resonant signal. Furthermore, the CARS signal has a nonlinear dependence on the number of probed molecular bonds. Nevertheless, the dual CARS measurements allowed for subtraction of the nonresonant background, resulting in a signal dominated by the resonant $\mathrm{CH}_{2}$ contribution.To compare the data from triglyceride analysis with data evaluated from the images, the background-corrected CARS signal of the lipid droplets was multiplied with the relative lipid area, resulting in the graph presented in Fig. 5(d). Comparing this CARS parameter for the two diets shows the same tendency as the triglyceride analysis, with higher lipid content for the HFSD-herring diet, for which a $22 \%$ higher median value is obtained.

To obtain additional qualitative information on the hepatic lipid content, CARS microspectroscopy measurements were carried out on two liver samples, one from each diet group, and the resulting spectra are shown in Fig. 6. The spectra show the signal in the region of hydrocarbon vibrations around $3000 \mathrm{~cm}^{-1}$, and both have the strongest signature located at $2850 \mathrm{~cm}^{-1}$ (indicated by the top arrow), corresponding to the symmetric stretch vibration of the $\mathrm{CH}_{2}$ groups in the lipid acyl chains. In addition to this peak, the spectra contain a broader feature between 2900 and $2950 \mathrm{~cm}^{-1}$. The ratio between the amplitude of the $2850-\mathrm{cm}^{-1}$ peak and the average signal in the 2900 - to $2950-\mathrm{cm}^{-1}$ region is higher for the spectrum of the HFSD-herring category, indicating longer acyl chains ${ }^{39}$ and resulting in more $\mathrm{CH}_{2}$ groups in turn giving coherent signal contributions to the resonance at $2850 \mathrm{~cm}^{-1}$. This interpretation is also supported by the higher ratio between the signal at $2850 \mathrm{~cm}^{-1}$ and the nonresonant background level measured at $2750 \mathrm{~cm}^{-1}$, compared to the HFSD-beef spectrum. In addition to the $\mathrm{CH}_{2}$ signal, the spectrum of the HFSD-herring sample shows an additional spectral line around $2880 \mathrm{~cm}^{-1}$ (indicated by the bottom arrow), corresponding to the asymmetric stretch vibration of $\mathrm{CH}_{2}$ groups. ${ }^{39-41}$ The presence of this peak indicates either longer acyl chains ${ }^{39}$ or reduction in interchain coupling. ${ }^{42}$ Bonn et al. have related the ratio between the CARS signals at 2880 and $2845 \mathrm{~cm}^{-1}$ to the degree of acyl chain ordering, and
Table 2 Molar percentages of saturated, monounsaturated, and polyunsaturated triglycerides for the HFSD-beef and HFSD-herring liver samples monitored by CARS microspectroscopy.

\begin{tabular}{|c|c|c|c|}
\hline \multicolumn{2}{|c|}{ Fatty acid } & \multirow{2}{*}{$\begin{array}{c}\text { HFSD herring } \\
2.41\end{array}$} & \multirow{2}{*}{$\frac{\text { HFSD beef }}{1.63}$} \\
\hline SFA & 14:0 & & \\
\hline & 15:0 & 0.44 & 0.33 \\
\hline & 16:0 & 32.70 & 29.95 \\
\hline & 18:0 & 3.75 & 1.22 \\
\hline & $20: 0$ & 0.23 & 0.16 \\
\hline & & 39.54 & 33.28 \\
\hline \multirow[t]{5}{*}{ MUFA } & $16: \ln 7$ & 5.25 & 8.91 \\
\hline & $18: \ln 7$ & 1.26 & 6.26 \\
\hline & $18: \ln 9$ & 27.96 & 47.26 \\
\hline & $20: \ln 9$ & 0.39 & 0.94 \\
\hline & & 34.85 & 63.36 \\
\hline \multirow[t]{5}{*}{ PUFA, $n-6$} & $18: 2 n 6$ & 4.82 & 2.70 \\
\hline & $18: 3 n 6$ & 0.00 & 0.00 \\
\hline & $20: 2 n 6$ & 0.00 & 0.00 \\
\hline & $20: 3 n 6$ & 0.00 & 0.09 \\
\hline & $20: 4 n 6$ & 0.76 & 0.21 \\
\hline \multirow[t]{4}{*}{ PUFA, $n-3$} & $20: 5 n 3$ & 5.54 & 0.06 \\
\hline & $22: 5 n 3$ & 1.54 & 0.11 \\
\hline & $22: 6 n 3$ & 11.56 & 0.18 \\
\hline & & 24.23 & 3.36 \\
\hline
\end{tabular}

found this parameter to be inversely related to the amount of double bonds, i.e., unsaturated lipids. ${ }^{43}$ Initially, this indicates a lower amount of unsaturated lipids for the HFSD herring. However, this is neither in agreement with the compositions of the diets nor with the triglyceride fatty acid compositions of the liver samples presented in Table 2. A higher amount of polyunsaturated fatty acids was found in the triglycerides of the HFSD-herring sample. However, this sample also showed an approximately ten times higher amount $(20.0 \%$ compared to $1.8 \%$ molar fractions) of long-acyl-chain $(n>=20)$ triglycerides, in agreement with the trend observed in the CARS spectra.

Although the multicomponent triglyceride composition increases the complexity of interpretation, the results clearly demonstrate that CARS microspectroscopy has the potential to provide not only morphological information on the lipid accumulation but also insights into the chemical composition of the lipid stores from spectral analysis. Multiplex CARS microscopy, generating spectra containing more data points than 

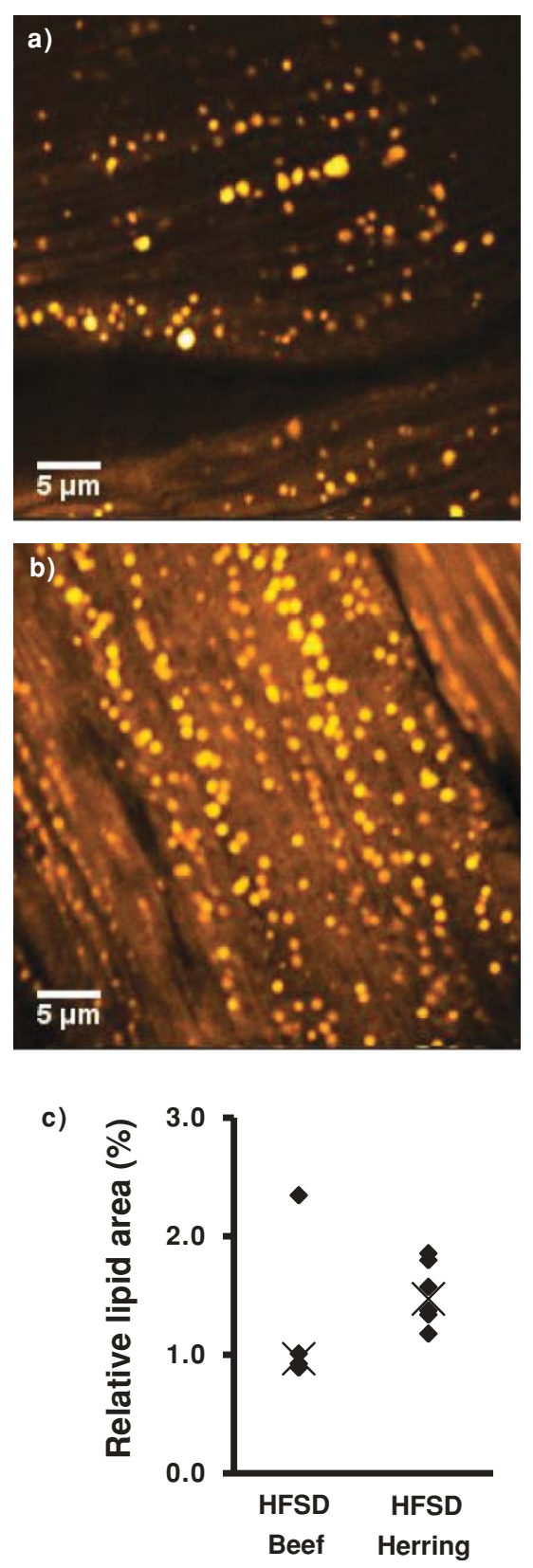

Fig. 7 CARS microscopy of diaphragm tissue from Ldlr-deficient mice fed (a) HFSD beef or (b) herring. Intramyocellular droplets can be observed in specimens from both diet groups, and comparison of the relative lipid areas shows a slightly higher amount of lipids for HFSDherring specimens in (c).

the presented excitation scans, and analysis methods that allow the pure spontaneous Raman spectrum to be extracted, ${ }^{41}$ can be combined with biochemical analysis to investigate liver lipid storage in more detail.

\subsection{Influence of Diet Composition on Intramyocellular Lipid Content}

The lipid content of the diaphragms of $L d l r$-deficient mice fed HFSD herring and beef were also investigated using CARS microscopy, and the results are shown in Fig. 7. Intramyocellular lipid droplets having diameters around $0.8 \mu \mathrm{m}$ could be identified for both diet groups, Figs. 7(a) and 7(b), and the lipid droplet
CARS signals were similar intensities for both categories, indicating droplets of comparable density. The lipid density, evaluated as a relative image area covered by lipid droplets, is shown in Fig. 7(c) and generally showed higher values for the HFSD herring, median value $1.5 \%$, than for the HFSDbeef specimens with median value $1.0 \%$. Sample images of low and high intramyocellular lipid storage are shown in Figs. 7(a) and 7(b), respectively.

\section{Conclusions}

Nonlinear microscopy has potential to serve as a powerful tool for characterization of biological tissue, demonstrated in this work by investigations of skeletal muscle and liver in mice fed different diets. Uptake and storage of lipids is related to many health problems and diseases, which makes label-free imaging of lipids in tissue by means of CARS microscopy of particular interest.

Although intramyocellular lipids recur more frequently in the skeletal muscle tissue of mice fed Western compared to standard diet, the main difference is observed between liver specimens of these categories. Comparing lipid storage in liver tissue for standard and Western diet shows extensive lipid accumulation for the Western diet case. In addition, these samples showed NAFLD, manifested by development of collagen fibers in the tissue and confirming the long-term detrimental effects of a high-fat diet.

The influence of lipid saturation level in Western-type diets was investigated for $L d l r$-deficient mice fed diets of different polyunsaturated lipid contents for 16 weeks. Biochemical analysis shows higher amounts of triglycerides and unsaturated lipids in HFSD-herring liver samples, and this is also supported by observations from CARS microscopy and microspectroscopy. Although these differences in overall lipid content and composition are observed, CARS microscopy data obtained from mice of both categories show hepatic lipid accumulation with large-sized structures, signifying NAFLD as a result of high-fat diet intake.

Of particular interest, especially apparent in the first study and in contrast to previous studies on NAFLD and steatohepatitis, is the relatively early detection of fibrous structures in liver following Western diet intake in our investigations. The high sensitivity of SHG microscopy in detecting collagen fibers demonstrates the potential of this method both as a tool for investigating early mechanisms that lead to fibrotic scarring of the liver, and for clinical diagnosis of steatohepatitis.

\section{Acknowledgments}

The excellent work by Robert Jakubowitz on preparing the mice samples is gratefully acknowledged by the authors. We are also grateful to Eva Jennische, Department of Biomedicine, University of Gothenburg, for fruitful discussions concerning the microscopy images and histological evaluations. The financial support from the Swedish Scientific Research Council is gratefully acknowledged.

\section{References}

1. N. A. v. Herpen and V. B. Schrauwen-Hinderling, "Lipid accumulation in non-adipose tissue and lipotoxicity," Physiol. Behav. 94(2), 231-241 (2008). 
2. G. Perseghin, P. Scifo, F. D. Cobelli, E. Pagliato, A. Battezzati, C. Arcelloni, A. Vanzulli, G. Testolin, G. Pozza, A. D. Maschio, and L. Luzi, "Intramyocellular triglyceride content is a determinant of in vivo insulin resistance in humans: a $1 \mathrm{H}-13 \mathrm{C}$ nuclear magnetic resonance spectroscopy assessment in offspring of type 2 diabetic parents," Diabetes 48(8), 1600-1606 (1999).

3. V. B. Schrauwen-Hinderling, M. K. C. Hesselink, P. Schrauwen, and M. E. Kooi, "Intramyocellular lipid content in human skeletal muscle," Obesity 14(3), 357-367 (2006).

4. L. Serfaty and M. Lemoine, "Definition and natural history of metabolic steatosis: clinical aspects of NAFLD, NASH and cirrhosis," Diabetes Metabol. 36(6), 634-637 (2008).

5. J. M. Clark, "The epidemiology of nonalcoholic fatty liver disease in adults," J. Clin. Gastroenterol. 40(Suppl 1), S5-S10 (2006).

6. G. C. Farrell and C. Z. Larter, "Nonalcoholic fatty liver disease: from steatosis to cirrhosis," Hepatology 43(2 Suppl 1), S99-S112 (2006).

7. C. F. A. Culling, Handbook of Histopathological and Histochemical Techniques, Butterworth, London (1974).

8. P. J. Campagnola and L. M. Loew, "Second-harmonic imaging microscopy for visualizing biomolecular arrays in cells, tissues and organisms," Nature Biotechnol. 21(11), 1356-1360 (2003).

9. P. J. Campagnola, A. C. Millard, M. Terasaki, P. E. Hoppe, C. J. Malone, and W. A. Mohler, "Three-dimensional high-resolution second-harmonic generation imaging of endogenous structural proteins in biological tissues," Biophys. J. 81, 493-508 (2002).

10. S. V. Plotnikov, A. C. Millard, P. J. Campagnola, and W. A. Mohler, "Characterization of the myosin-based source for second-harmonic generation from muscle sarcomeres," Biophys. J. 90, 693-703 (2006).

11. S. V. Plotnikov, A. M. Kenny, S. J. Walsh, B. Zubrowski, C. Joseph, V. L. Scranton, G. A. Kuchel, D. Dauser, M. Xu, C. C. Pilbeam, D. J. Adams, R. P. Dougherty, P. J. Campagnola, and W. A. Mohler, "Measurement of muscle disease by quantitative second-harmonic generation imaging," J. Biomedi. Opt. 13(4), 044018 (2008).

12. M. Banavar, E. P. W. Kable, F. Braet, X. M. Wang, M. D. Gorrell, and G. Cox, "Detection of collagen by second harmonic microscopy as a diagnostic tool for liver fibrosis," Proceedings of SPIE 6089, 60891B (2006).

13. D. C. S. Tai, N. Tan, S. Xu, C. H. Kang, S. M. Chia, C. L. Cheng, A. Wee, C. L. Wei, A. M. Raja, G. Xiao, S. Chang, J. C. Rajapakse, P. T. C. So, H. H. Tang, C. S. Chen, and H. Yu, "Fibro-C-Index: comprehensive, morphology-based quantification of liver fibrosis using second harmonic generation and two-photon microscopy," J. Biomed. Opt. 14(4), 044013 (2009).

14. J. X. Cheng and X. S. Xie, "Coherent anti-Stokes Raman scattering microscopy: instrumentation, theory, and applications," J. Phys. Chem. $B$ 108, 827-840 (2004).

15. J. X. Cheng, "Coherent anti-Stokes Raman scattering microscopy," Appl. Spectrosc. 91, 197-208 (2007).

16. C. Brackmann, J. Norbeck, M. Åkeson, D. Bosch, C. Larsson, L. Gustafsson, and A. Enejder, "CARS microscopy of lipid stores in yeast: the impact of nutritional state and genetic background " J. Raman Spectrosc. 40(7), 748-756 (2009).

17. C. Heinrich, A. Hofer, A. Ritsch, C. Ciardi, S. Bernet, and M. RitschMarte, "Selective imaging of saturated and unsaturated lipids by widefield CARS-microscopy," Opt. Express 16(4), 2699-2708 (2008).

18. T. Hellerer, C. Axäng, C. Brackmann, P. Hillertz, M. Pilon, and A. Enejder, "Monitoring of lipid storage in Caenorhabditis elegans using coherent anti-Stokes Raman scattering (CARS) microscopy," Proc. Nat. Acad. Sci. 104, 14658-14663 (2007).

19. J. Zhu, B. Lee, K. K. Buhman, and J. X. Cheng, "A dynamic, cytoplasmic triacylglycerol pool in enterocytes revealed by ex vivo and in vivo coherent anti-Stokes Raman scattering imaging," J. Lipid Res. 50, 1080-1089 (2009).

20. Y. M. Wu, H. C. Chien, W. T. Chang, J. W. Jahn, H. L. Lin, and I. Liau, "Quantitative assessment of hepatic fat of intact liver tissues with coherent anti-Stokes Raman scattering microscopy," Anal. Chem. 81(4), 1496-1504 (2009).

21. G. S. Getz and C. A. Reardon, "Diet and murine atherosclerosis," Arterioscler. Thromb. Vascular Biol. 26(2), 242-249 (2006).

22. O. Burkacky, A. Zumbusch, C. Brackmann, and A. Enejder, "Dualpump coherent anti-Stokes Raman scattering microscopy," Opt. Lett. 31(24), 3656-3658 (2006).
23. M. D. Abramoff, P. J. Magelhaes, and S. J. Ram, "Image processing with ImageJ," Biophotonics International 11(7), 36-42 (2004).

24. M. H. Ross, E. J. Reith, and L. J. Romrell, Histology a Text and Atlas, Williams and Wilkins, Baltimore, MD (1989).

25. H. Hoppeler, P. Lüthi, H. Claassen, E. R. Weibel, and H. Howald, "The ultrastructure of the normal human skeletal muscle," Pfiügers Archiv Eur. J. Physiol. 344(3), 217-232 (1973).

26. C. S. Shaw, D. A. Jones, and A. J. Wagenmakers, "Network distribution of mitochondria and lipid droplets in human muscle fibres," Histochem. Cell Biol. 129(1), 65-72 (2008).

27. C. S. Shaw, M. Sherlock, P. M. Stewart, and A. J. Wagenmakers, "Adipophilin distribution and colocalization with lipid droplets in skeletal muscle," Histochem. Cell Biol. 131(5), 575-581 (2009).

28. K. E. Shortreed, M. P. Krause, J. H. Huang, D. Dhanani, J. Moradi, R. B. Ceddia, and T. J. Hawke, "Muscle-specific adaptations, impaired oxidative capacity and maintenance of contractile function characterize diet-induced obese mouse skeletal muscle," PloS One 4(10), e7293 (2009).

29. J. D. Bancroft and M. Gamble, Theory and Practice of Histological Techniques, Churchill Livingstone, Elsevier, Amsterdam (2002).

30. C. Z. Larter and M. M. Yeh, "Animal models of NASH: Getting both pathology and metabolic context right," J. Gastroenterol. Hepatol. 23, 1635-1648 (2008).

31. L. D. DeLeve, X. Wang, G. C. Kanel, R. D. Atkinson, and R. S. McCuskey, "Prevention of hepatic fibrosis in a murine model of meatbolic syndrome with nonalcoholic steatohepatitis," Am. J. Pathol. 173(4), 993-1001 (2008).

32. G. Cox, E. Kable, A. Jones, I. Fraser, F. Manconi, and M. Gorrell, "3-dimensional imaging of collagen using second harmonic generation," J. Structur. Biol. 141(1), 53-62 (2003).

33. H. Bao, A. Boussioutas, R. Jeremy, S. Russell, and M. Gu, "Second harmonic generation imaging via nonlinear endomicroscopy," Opt. Express 18(2), 1255-1260 (2009).

34. Y. Wu, Y. Leng, J. Xi, and X. Li, "Scanning all-fiber-optic endomicroscopy system for 3D nonlinear optical imaging of biological tissues," Opt. Express 17(10), 7907-7915 (2009).

35. L. A. Videla, R. Rodrigo, J. Araya, and J. Poniachik, "Oxidative stress and depletion of hepatic long-chain polyunsaturated fatty acids may contribute to nonalcoholic fatty liver disease," Free Rad. Biol. Med. 37(9), 1499-1507 (2004).

36. J. Araya, R. Rodrigo, L. A. Videla, L. Thielemann, M. Orellana, P. Pettinelli, and J. Poniachik, "Increase in long-chain polyunsaturated fatty acid $n-6 / n-3$ ratio in relation to hepatic steatosis in patients with non-alcoholic fatty liver disease," Clin. Sci. 106(6), 635-643 (2004).

37. P. Pettinelli, T. Del Pozo, J. Araya, R. Rodrigo, A. V. Araya, G. Smok, A. Csendes, L. Gutierrez, J. Rojas, O. Korn, F. Maluenda, J. C. Diaz, G. Rencoret, I. Braghetto, J. Castillo, J. Poniachik, and L. A. Videla, "Enhancement in liver SREBP-1c/PPAR-alpha ratio and steatosis in obese patients: correlations with insulin resistance and n-3 long-chain polyunsaturated fatty acid depletion," Biochim. Biophys. Acta 1792(11), 1080-1086 (2009).

38. R. B. Alfin-Slater, S. Bernick, "Changes in tissue lipids and tissue histology resulting from essential fatty acid deficiency in rats," $\mathrm{Am} \mathrm{J}$ Clin Nutr. 6(6), 613-624 (1958).

39. S. Bresson, M. El Marssi, and B. Khelifa, "Raman spectroscopy investigation of various saturated monoacid triglycerides," Chem. Phys. Lipids 134, 119-129 (2005).

40. R. G. Snyder, S. L. Hsu, and S. Krimm, "Vibrational spectra in the C-H stretching region and the structure of the polymethylene chain," Spectrochim. Acta 34, 395-406 (1978).

41. H. A. Rinia, M. Bonn, M. Müller, and E. M. Vartiainen, "Quantitative CARS spectroscopy using the maximum entropy method: the main lipid phase transition," ChemPhysChem 8(2), 279-287 (2007).

42. H. A. Rinia, K. N. J. Burger, M. Bonn, and M. Müller, "Quantitative label-free imaging of lipid composition and packing of individual cellular lipid droplets using multiplex CARS microscopy," Biophys. J. 95, 4908-4914 (2008).

43. M. Bonn, M. Müller, H. A. Rinia, and K. N. J. Burger, "Imaging of chemical and physical state of inidividual cellular lipid droplets using multiplex CARS microscopy," J. Raman Spectrosc. 40, 763-769 (2009). 